Comment. Math. Helv. 75 (2000) 353-367

(C) 2000 Birkhäuser Verlag, Basel

$0010-2571 / 00 / 030353-15 \$ 1.50+0.20 / 0$

Commentarii Mathematici Helvetici

\title{
Additivity of tunnel number for small knots
}

\author{
Jennifer Schultens
}

\begin{abstract}
We show that for small knots $K_{1}, K_{2}, t\left(K_{1}\right)+t\left(K_{2}\right)-1 \leq t\left(K_{1} \# K_{2}\right) \leq t\left(K_{1}\right)+$ $t\left(K_{2}\right)+1$, and that for small knots $K_{1}, \ldots, K_{n}, t\left(K_{1} \# \ldots \# K_{n}\right) \geq n$.
\end{abstract}

Mathematics Subject Classification (2000). 57N10.

Keywords. Small knot, tunnel number, decomposing annulus.

\section{Introduction}

The tunnel number of a knot has been shown to behave quite erratically under the operation of connected sum. On the one hand, Kobayashi has exhibited knots for which the tunnel number degenerates by an arbitrarily high number under connected sum ([6]) and on the other hand Moriah and Rubinstein and independently Morimoto, Sakuma and Yokota have exhibited knots for which the tunnel number is strictly super-additive under connected sum ([8] and [10]). Restricting attention to connected sums of small knots circumvents some of the possibilities and many of the technical difficulties encountered in the work of Kobayashi concerning torus decompositions of manifolds and of Morimoto concerning the additivity of the tunnel numbers of knots ([7] and [9]). The more general question of how Heegaard genus behaves when two manifolds are glued together along an annulus remains open. This project was inspired by and answers problem 1.70 in [5] for the case of small knots. I wish to thank Andrew Casson and Marty Scharlemann for helpful conversations. I also wish to thank MSRI, where part of this research was carried out.

\section{Definitions and general facts}

Definition 1. For any submanifold $L$ of $M, \eta(L)$ denotes an open regular neighborhood of $L$ in $M$ and $N(L)$ denotes a closed regular neighborhood of $L$ in $M$.

Research supported by an NSF postdoctoral fellowship 
Definition 2. Let $K$ be a knot in $S^{3}$, then $C(K)=S^{3}-\eta(K)$.

Definition 3. A knot $K$ is small, if $C(K)$ contains no closed essential surfaces.

Definition 4. A tunnel system for a knot $K$ is a collection of disjoint arcs $\mathcal{T}=t_{1}$ $\cup \ldots \cup t_{n}$ embedded in $C(K)$ such that $C(K)-\eta(\mathcal{T})$ is a handlebody. We require one endpoint of $t_{i}$ to lie on $\partial C(K)$ and the other to either also lie on $\partial C(K)$, or to meet an interior point of $t_{i}$. The tunnel number of $K$, denoted by $t(K)$, is the least number of arcs required in a tunnel system for $K$.

Definition 5. A compression body is a 3-manifold $W$ obtained from a closed orientable surface $F$ by attaching 2-handles to $F \times\{0\} \subset F \times I$ and capping off any resulting 2-sphere boundary components. We denote $F \times\{1\}$ by $\partial_{+} W$ and $\partial W-$ $\partial_{+} W$ by $\partial_{-} W$.

Definition 6. A spine $X$ of a compression body $W$ is a properly embedded 1complex in $W$ such that $X \cup \partial_{-} W$ is connected and such that $W$ collapses to $X$ $\cup \partial_{-} W$.

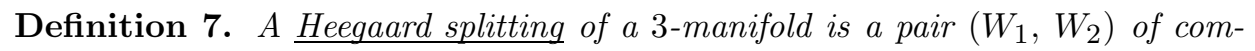
pression bodies, such that $W_{1} \cap W_{2}=\partial_{+} W_{1}=\partial_{+} W_{2}$ and $M=W_{1} \cup W_{2}$. We call $\partial_{+} W_{1}=\partial_{+} W_{2}$ the splitting surface or Heeqaard surface and denote it by $F$.

Definition 8. $A$ disk $D$ in a compression body $W$ is an essential disk if $(D, \partial D) \subset$ $\left(W, \partial_{+} W\right)$ and $\partial D$ is an essential curve in $\partial_{+} W$. A defining collection of disks for $\underline{W}$ is a collection of essential disks $\mathcal{D}=D_{1} \cup \ldots \cup D_{n}$ which cut $W$ into $\partial_{-} W$ $\cup 3$-balls.

Definition 9. A Heegaard splitting is (weakly) reducible if there are essential disks $D_{1}$ and $D_{2}$, such that $\left(D_{i}, \partial D_{i}\right) \subset\left(W_{i}, \partial_{+} W_{i}\right)$ and $\left(\partial D_{1} \cap \partial D_{2}=\emptyset\right) \partial D_{1}$ $=\partial D_{2}$. A Heegaard splitting which is not weakly reducible is strongly irreducible.

Definition 10. Let $K_{1} \# K_{2}$ be the connected sum of the knots $K_{1}$ and $K_{2}$. Then there is a sphere $S$ which intersects $K_{1} \# K_{2}$ in exactly two points, the annulus $A=S \cap C\left(K_{1} \# K_{2}\right)$ is called a decomposing annulus. Note that cutting $C\left(K_{1}\right.$ \# $K_{2}$ ) along $A$ produces a copy of $C\left(K_{1}\right)$ and of $C\left(K_{2}\right)$.

The following four lemmata are well known but crucial. We repeat them here for completeness.

Lemma 1. An incompressible and boundary incompressible surface $S$ with $\partial S \neq$ $\emptyset$ properly embedded in a compression body $W$ is either an essential disk, or an annulus with one boundary component in $\partial_{-} W$ and the other in $\partial_{+} W$. 
Proof. This is [1, Lemma 9].

Lemma 2. An incompressible surface $S$ in a compression body $W$ with $\partial S \subset$ $\partial_{+} W$ cuts $W$ into compression bodies.

Proof. Let $\mathcal{D}$ be a defining collection of disks for $W$. Isotope $S$ so that $\#|\mathcal{D} \cap S|$ is minimal. Then, in particular, $\mathcal{D} \cap S$ contains no simple closed curves. Cut $W$ along $\mathcal{D}$ to obtain $\left(\partial_{-} W \times I\right) \cup(3-$ balls $)$. Since $\partial S \subset \partial_{+} W$, Lemma 1 implies that $S$ is boundary parallel in or disjoint from any given component of $\left(\partial_{-} W\right.$ $\times I)$ and hence cuts it into (closed surface) $\times I$ and perhaps handlebodies. Furthermore, the incompressibility of $S$ guarantees that $S$ intersects each 3-ball in meridian disks. Therefore the closure of a component of $W-S$ is obtained by attaching (closed surface) $\times I$ components, handlebodies and 3-balls along disks. The result is a compression body.

Lemma 3. Let $M^{1}$ and $M^{2}$ be 3-manifolds such that $M^{i}$ has a Heegaard splitting $\left(V_{1}^{i}, V_{2}^{i}\right)$ of genus $g^{i}$ and there is an annulus $A^{i}$ in $\partial M^{i}$. Then the manifold $M$ obtained by glueing $M^{1}$ and $M^{2}$ together along $A^{1}$ and $A^{2}$ has a Heegaard splitting of genus $g=g^{1}+g^{2}$.

Proof. Let $B^{i}$ be the boundary component of $M^{i}$ containing $A^{i}$. We may assume that $B^{i} \subset V_{1}^{i}$. Then by shrinking $V_{1}^{i}$, we may assume that $V_{1}^{i}$ consists of a collar of $B^{i}$ in $M^{i}$, denoted by collar $\left(B^{i}\right)$, 1-handles, some of which are attached to collar $\left(B^{i}\right)$ and, perhaps, other collars of boundary components of $M^{i}$.

Let $\alpha^{i}$ be a simple arc in $A^{i}$ connecting the two components of $\partial A^{i}$ and let $D^{i}$ $=A^{i}-\eta\left(\alpha^{i} \cup \partial A^{i}\right)$. Let collar $\left(D^{i}\right)$ be the subset of $\operatorname{collar}\left(B^{i}\right)$ which is a collar on $D^{i}$. We may assume that collar $\left(D^{i}\right)$ does not meet any 1-handles. Glue $M^{1}$ to $M^{2}$ along $A^{1}$ and $A^{2}$ so that $D^{1}$ and $D^{2}$ match up. Define $V_{1}=\left(V_{1}^{1}-\operatorname{collar}\left(D^{1}\right)\right)$ $\cup\left(V_{1}^{2}-\operatorname{collar}\left(D^{2}\right)\right)$ and $V_{2}=\left(V_{2}^{1} \cup \operatorname{collar}\left(D^{1}\right)\right) \cup\left(V_{2}^{2} \cup \operatorname{collar}\left(D^{2}\right)\right)$. Then $\left(V_{1}, V_{2}\right)$ is a Heegaard splitting of the manifold $M$ obtained by glueing $M^{1}$ and $M^{2}$ together along $A^{1}$ and $A^{2}$ and has genus $g=g^{1}+g^{2}$.

Definition 11. Suppose an arc $\alpha$ shares an endpoint with an arc $\beta$. An arc slide of $\alpha$ over $\beta$ is the result of replacing $\alpha$ with $\overline{\alpha \cup \beta}$, where $\overline{\alpha \cup \beta}$ is a pushoff of $\alpha \cup \beta$.

Remark 4. Tunnel systems of a knot correspond to Heegaard splittings of the complement of the knot. Given a tunnel system $\mathcal{T}$ for $K$, set $V_{1}=N(\partial C(K)$ $\cup \mathcal{T})$ and $V_{2}$ equal to the closure of $C(K)-V_{1}$. Conversely, given a Heegaard splitting $\left(V_{1}, V_{2}\right)$ of $C(K)$, we may assume that $\partial C(K) \subset V_{1}$. Then, after arc slides, if necessary, a spine $X_{1}$ of $V_{1}$ is a tunnel system for $K$. Note that in this correspondence, the number of $\operatorname{arcs}$ in $\mathcal{T}$ is exactly one less than the genus of ( $V_{1}$, $\left.V_{2}\right)$. 
Corollary 5. $t\left(K_{1} \# K_{2}\right) \leq t\left(K_{1}\right)+t\left(K_{2}\right)+1$.

Proof. This follows from Lemma 3 and Remark 4.

Lemma 6. The splitting surface $F$ of a strongly irreducible Heegaard splitting $\left(V_{1}\right.$, $V_{2}$ ) of $M$ may be isotoped to intersect a properly embedded incompressible surface $S \subset M$ only in essential simple closed curves.

Proof. Let $X_{i}$ be a spine of $V_{i}$. If $X_{i}$ is disjoint from $F$, then, perhaps after isotopy, $F \cap S=\emptyset$. So suppose that $X_{i} \cap S \neq \emptyset$, for $i=1,2$. Let $H: F \times I \rightarrow M-\eta\left(X_{1}\right.$ $\left.\sqcup X_{2}\right)$ be a foliation, by surfaces isotopic to $F$, of $M-\eta\left(X_{1} \sqcup X_{2}\right)$. We denote $H(F, t)$ by $F_{t}$ and assume that $F_{i}=\partial N\left(X_{i}\right)$.

Set $\mathcal{V}_{i}=\left\{t \mid F_{t} \cap S\right.$ contains simple closed curves bounding disks entirely in $V_{i} \cap S$ \}. Then for $t$ close to $1, t \in \mathcal{V}_{1}$, whereas for $t$ close to $2, t \in \mathcal{V}_{2}$. To better understand $\mathcal{V}_{1}$ and $\mathcal{V}_{2}$, consider the singular foliation of $S-\eta\left(X_{1}\right.$ $\sqcup X_{2}$ ) induced by $H$. If a regular leaf contains a simple closed curve bounding disks entirely in $V_{i}$, then so do nearby regular leaves. Furthermore, if the regular leaves limiting on a singular leaf $\sigma$ from one side all contain simple closed curves bounding disks entirely in $V_{i}$, then so does $\sigma$. It follows that either $\mathcal{V}_{1} \cap \mathcal{V}_{2} \neq \emptyset$ or the complement of $\mathcal{V}_{1} \cup \mathcal{V}_{2}$ has nonempty interior. Since $\left(V_{1}, V_{2}\right)$ is strongly irreducible, the latter must be the case. Choose $t$ in the complement of $\mathcal{V}_{1} \cup \mathcal{V}_{2}$ and so that $F_{t} \cap S$ is a regular leaf. Then $F_{t}$ is a copy of $F$ which intersects $S$ only in essential simple closed curves.

\section{Tunnel systems corresponding to weakly reducible Heegaard splittings}

Tunnel systems fall into two types, those corresponding to weakly reducible Heegaard splittings and those corresponding to strongly irreducible Heegaard splittings. We here investigate tunnel systems for $K_{1} \# K_{2}$ which correspond to weakly reducible Heegaard splittings and show how they define tunnel systems for $K_{1}$ and $K_{2}$. We exploit the ideas in introduced in [2] and extended in [13] and [12], linking weakly reducible Heegaard splittings and incompressible surfaces.

Definition 12. Let $\left(V_{1}, V_{2}\right)$ be an irreducible Heegaard splitting of $M$. We may think of $M$ as being obtained from $\partial_{-} V_{1} \times I$ by attaching all 1-handles in $V_{1}$ followed by all 2-handles dual to 1-handles in $V_{2}$, followed, perhaps, by 3-handles. An untelescoping of $\left(V_{1}, V_{2}\right)$ is a rearrangement of the order in which the 1-handles (of $V_{1}$ ) and the 2-handles (dual to the 1-handles of $V_{2}$ ) are attached, so that $M$ is decomposed into submanifolds $M^{0}, \ldots, M^{k}$, meeting along surfaces $S^{1}, \ldots$, $S^{k}$, which are incompressible in $M$, and which inherit, from a subcollection of the original 1-handles and 2-handles, strongly irreducible Heegaard splittings $\left(V_{1}^{0}\right.$, 


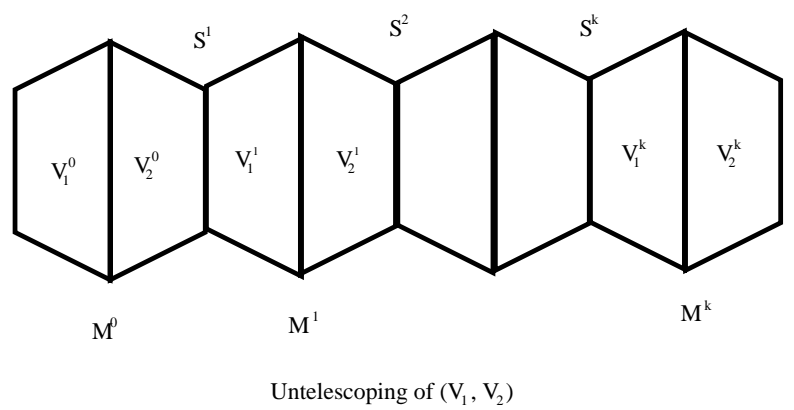

Figure 1.

$\left.V_{2}^{0}\right), \ldots,\left(V_{1}^{k}, V_{2}^{k}\right)$. For details see [13] and [12].

Remark 7. The proof of the Main Theorem in [2] shows that if $\left(V_{1}, V_{2}\right)$ is irreducible but weakly reducible, then $k \geq 1$ in any untelescoping of $\left(V_{1}, V_{2}\right)$.

Remark 7 shows that we must understand what sort of incompressible surfaces occur in the complement of the connected sum of small knots.

Lemma 8. Let $S$ be a separating essential surface in $C\left(K_{1} \# K_{2}\right)$, where $K_{i}$ is a small prime knot. Then $S \subset C\left(K_{i}\right)$ for either $i=1$ or $i=2$; and $S$ is boundary parallel in $C\left(K_{i}\right)$. (See fig. 7)

Definition 13. A surface of the type described is called a swallow follow torus.

Proof. Let $A$ be the decomposing annulus. If $S \cap A=\emptyset$, then the claim follows. So suppose $S \cap A \neq \emptyset$, and set $S^{i}=S \cap C\left(K_{i}\right)$. Here $C\left(K_{i}\right)$ is obtained from $C\left(K_{1} \# K_{2}\right)$ by cutting along the decomposing annulus. Since $\partial S^{i}$ consists of meridians, the meridian is a boundary slope. Hence by [3, Theorem 2.0.3], $C\left(K_{i}\right)$ contains a closed essential surface. But this contradicts the fact that $C\left(K_{i}\right)$ is small.

Theorem 9. Let $K_{1}, K_{2}$ be small knots, and suppose the tunnel system $\mathcal{T}$ realizing the tunnel number of $K_{1} \# K_{2}$ corresponds to a weakly reducible Heegaard splitting. Then $t\left(K_{1}\right)+t\left(K_{2}\right) \leq t\left(K_{1} \# K_{2}\right)$.

Proof. Let $\left(V_{1}, V_{2}\right)$ be the Heegaard splitting corresponding to $\mathcal{T}$. Since $\mathcal{T}$ realizes the tunnel number of $K_{1} \# K_{2},\left(V_{1}, V_{2}\right)$ is irreducible. Consider an untelescoping of $\left(V_{1}, V_{2}\right)$. By Remark $7, k \geq 1$. If an $S^{i}$ were boundary parallel in $C\left(K_{1} \#\right.$ $\left.K_{2}\right)$, then the untelescoping of $\left(V_{1}, V_{2}\right)$ would define a Heegaard splitting of $C\left(K_{1}\right.$ $\# K_{2}$ ) of lower genus, contradicting the choice of $\mathcal{T}$. Hence each $S^{i}$ must be a swallow follow torus. Since the two distinct isotopy classes of swallow follow tori 


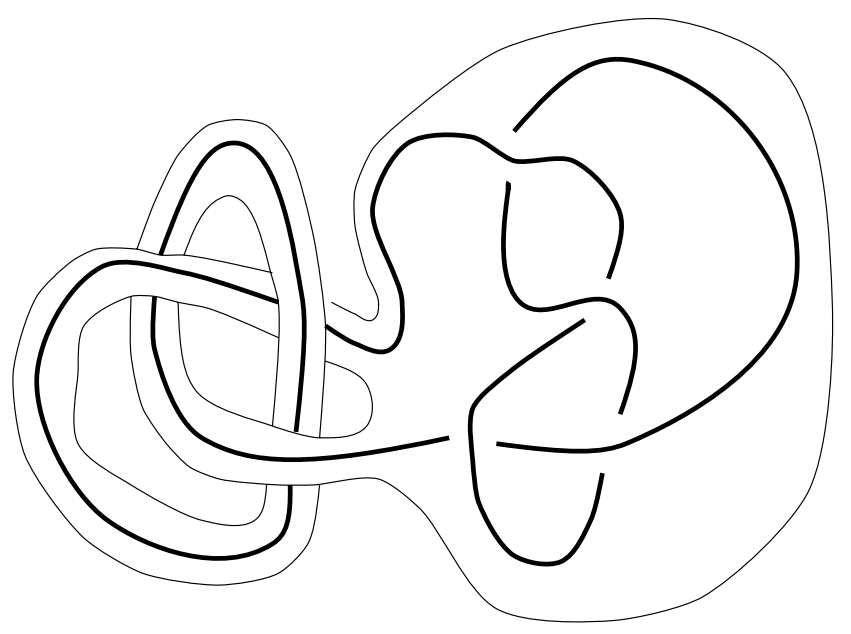

A swallow-follow torus

Figure 2.

can't be embedded simultaneously, the $S^{i}$ must in fact be parallel swallow follow tori.

If we assume, for convenience of notation, that $S^{i} \subset C\left(K_{1}\right)$ for $i=1, \ldots, k$, then $M_{0}$ is homeomorphic to $C\left(K_{1}\right)$ minus a 2-handle, hence $\left(V_{1}^{0}, V_{2}^{0}\right)$ defines a Heegaard splitting for $C\left(K_{1}\right)$, and $M^{k}$ is homeomorphic to $C\left(K_{2}\right)$, hence $\left(V_{1}^{k}\right.$, $\left.V_{2}^{k}\right)$ defines a Heegaard splitting for $C\left(K_{2}\right)$. Counting the 1-handles in $V_{1}^{0}$ and $V_{1}^{k}$, which is the number of arcs in the corresponding tunnel system, shows that $t\left(K_{1}\right)+t\left(K_{2}\right) \leq t\left(K_{1} \# K_{2}\right)$.

Remark 10. By Lemma $3, k \leq 2$. For more subtle reasons (certain properties of the Heegaard splittings $\left(V_{1}^{i}, V_{2}^{i}\right)$ and of Heegaard splittings of $($ surface $\left.) \times I\right), k$ $=1$.

\section{Tunnel systems corresponding to strongly irreducible Hee- gaard splittings}

In this section we restrict our attention to tunnel systems of knots which correspond to strongly irreducible Heegaard splittings. Most importantly, we show how to use a tunnel system for a connected sum of knots to construct tunnel systems for its summands when all tunnel systems realizing the tunnel number of the connected sum of knots correspond to strongly irreducible Heegaard splittings. The trick in Definition 14 allows us to choose the original tunnel system so that this process proves bounded degeneration of tunnel number under connected sum. 
The results were originally inspired by [9]. However, the arguments changed dramatically due to the use of the notion of strongly irreducible Heegaard splittings and the terminology and techniques available for their study.

Theorem 9 allows us to make certain assumptions. Since these assumptions will be used over and over again, we summarize them here:

Assumption 1: All tunnel systems realizing $t\left(K_{1} \# K_{2}\right)$ correspond to strongly irreducible Heegaard splittings.

Assumption 2: The tunnel system $\mathcal{T}$ realizing $t\left(K_{1} \# K_{2}\right)$ is chosen so that the splitting surface of the corresponding Heegaard splitting may be isotoped to intersect the decomposing annulus in the least number of essential curves.

The following definition gives an operation which helps move arcs in a tunnel system away from a decomposing annulus. The trick is due to Marty Scharlemann.

Definition 14. Let $\mathcal{T}$ be a tunnel system for the knot $K$, let $\left(V_{1}, V_{2}\right)$ be the corresponding Heegaard splitting of $C(K)$ and suppose there is an annulus $\tilde{A}$ with interior $(\tilde{A}) \subset V_{2}$, such that one component, $\partial_{1} \tilde{A}$, of $\partial \tilde{A}$ lies on $\partial V_{1}$ and intersects the boundary of a meridian disk $D$ of $V_{1}$ exactly once and such that the other component, $\partial_{2} \tilde{A}$, of $\partial \tilde{A}$ lies on the boundary of an arbitrarily small regular neighborhood of $\partial C(K)$. Then setting $\tilde{V}_{1}=N(\partial C(K) \cup \mathcal{T} \cup \tilde{A})$ and $\tilde{V}_{2}$ the closure of the complement produces a Heegaard splitting of $C(K)$. To see that $\tilde{V}_{1}$ is a compression body, consider a collection $\mathcal{D}_{1}$ of defining disks for $V_{1}$ containing $D$. By [16, Lemma 2.1] $N\left(D \cup \partial_{1} \tilde{A}\right)$ is a solid torus summand of $V_{1}$. Let $\tilde{D}$ be an essential disk in $V_{1}$ which cuts off this solid torus summand. Then $\left(\mathcal{D}_{1}-D\right) \cup$ $\tilde{D}$ is a defining collection of disks for $\tilde{V}_{1} . \tilde{V}_{2}$ is a handlebody by the proof of [1, Lemma 9]). This Heegaard splitting has the same genus as the Heegaard splitting corresponding to $\mathcal{T}$. (For the splitting surface of the former is obtained by cutting the splitting surface of the latter along two essential curves and reconnecting the resulting boundary components along annuli, this operation doesn't change the Euler characteristic of the surface.) The new Heegaard splitting corresponds to a new tunnel system $\mathcal{T}^{\prime}$. We will say that $\mathcal{T}^{\prime}$ is obtained from $\mathcal{T}$ by replacing one of the arcs in $\mathcal{T}$ by its dual. (The terminology generalizes that used in the case where the tunnel system contains only one arc.)

Lemma 11. Let $K_{1}$ and $K_{2}$ be small knots. Suppose that $K_{1} \# K_{2}$ satisfies Assumption 1, and the tunnel system $\mathcal{T}$ for $K_{1} \# K_{2}$ satisfies Assumption 2. Then, after isotopy, the splitting surface $F$ of the Heegaard splitting $\left(V_{1}, V_{2}\right)$ corresponding to $\mathcal{T}$ intersects $A$ only in essential curves, and $\#|F \cap A| \leq 4$.

Proof. Suppose that $F \cap A$ consists only of essential curves and that $\#|F \cap A|$ $>4$. Then one of the components of $V_{1} \cap A$ is boundary reducible in $V_{1}$. We may assume, by choosing an outermost one, that the boundary reducing disk is disjoint from $A$. Then after performing the boundary compression along this disk, $V_{1} \cap A$ 


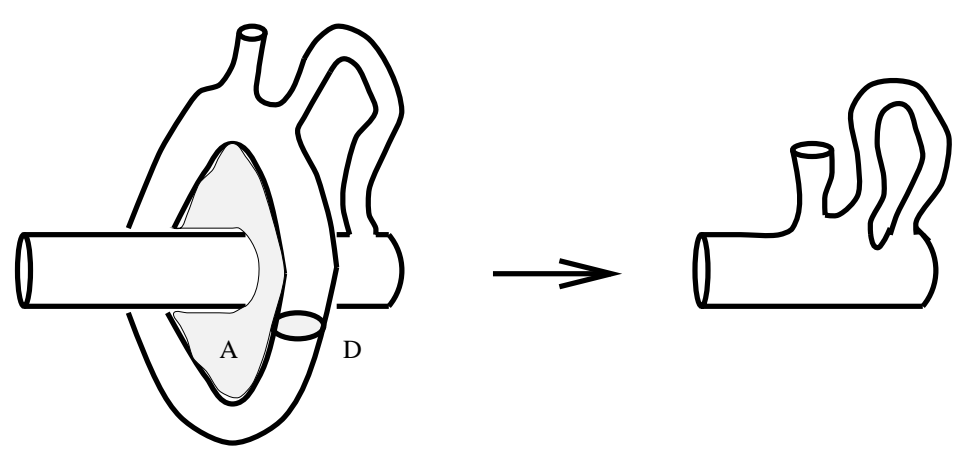

Replacing an arc by its dual

Figure 3.

consists of one disk and at least three annuli. Perform an ambient 2-surgery on $F$ along the disk, and then perform ambient 2-surgery on the resulting surface,along all compressing disks disjoint from $A$, to obtain a surface $F^{*}$, each component of which is separating. We will denote the two sides of $F^{*}$ by $V_{1}^{*}$ and $V_{2}^{*}$. A priori, neither $V_{1}^{*}$ nor $V_{2}^{*}$ need be connected.

Note that the ambient 2-surgeries may be performed in sequence. Then at each step, the next compressing disk $E$ can be isotoped so that $\partial E$ lies entirely in $F$. Consider the annulus in $F$ consisting of a bicollar of $\partial E$. It follows from [14, Lemma 2.6] and the strong irreducibility of $\left(V_{1}, V_{2}\right)$, that $E$ lies either entirely in $V_{1}$ or entirely in $V_{2}$. The strong irreducibility further implies, that in this case, $E$ lies entirely in $V_{1}$. It follows that $V_{1}^{*} \subset V_{1}$ (whereas $V_{2}^{*}=V_{2} \cup 2$-handles). In particular, $V_{1}^{*}$ needn't be connected, but $V_{2}^{*}$ must be.

Consider a component $\tilde{F}^{*}$ of $F^{*} \cap C\left(K_{i}\right)$. If $\tilde{F}^{*}$ were an essential surface in $C\left(K_{i}\right)$, then the meridian of $C\left(K_{i}\right)$ would be a boundary slope, hence by [3, Theorem 2.0.3], $C\left(K_{i}\right)$ would contain a closed essential surface. Since $K_{i}$ is small, and since $\tilde{F}^{*}$ is incompressible in $C\left(K_{i}\right), \tilde{F}^{*}$ must be boundary parallel in $C\left(K_{i}\right)$. Hence $\tilde{F}^{*}$ is either parallel into a subannulus of $A$, or into $\partial C\left(K_{i}\right) \cup$ 2 subannuli of $A$. In the former case, we will call $\tilde{F}^{*}$ a narrow annulus, and in the latter case a wide annulus. Note that since a component of $F^{*}$ can be constructed by identifying annuli along their boundary, it must be a torus.

Claim 1: A narrow annulus in $F^{*}$ cobounds, together with a subannulus of $A$, a solid torus entirely in $V_{1}^{*}$.

Suppose the solid torus $T$ cobounded by a narrow annulus $N_{1}$ in $F^{*}$ and a subannulus $A^{\prime}$ of $A$ meets the interior of $V_{2}^{*}$. Then we may assume, by replacing $N_{1}$ with a narrow annulus properly embedded in $T$ if necessary, that a collar of $N_{1}$ in $T$ lies in $V_{2}^{*}$. The torus $T$ defines an isotopy of $N_{1}$ into $A^{\prime}$. Since $V_{2}^{*}=$ $V_{2} \cup 2$-handles, that is, since $V_{2}^{*}$ contains $V_{2}$, this isotopy defines an isotopy of $F$ 


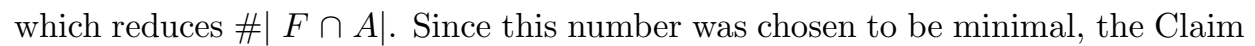
follows.

Claim 2: There can be at most two nested components of $F^{*}$.

This follows from the fact that (the "side") $V_{2}^{*}$ (of $F^{*}$ ) is connected.

Claim 3: There is exactly one component of $F^{*}$ which is parallel into $\partial C\left(K_{1} \#\right.$ $\left.K_{2}\right)$.

By Claim 2, there can be no more than two such components. If there were exactly two such components, then, again by Claim $2, V_{1}^{*} \subset V_{1}$ would contain a copy of $C\left(K_{1} \# K_{2}\right)$, but this is impossible. If there were no such component, then the component $C$ of $V_{1}^{*}$ containing $\partial C\left(K_{1} \# K_{2}\right)$ would be cobounded by tori in $F^{*}$ none of which was parallel to $\partial C\left(K_{1} \# K_{2}\right)$. Since $V_{1}^{*}$ is obtained by cutting $V_{1}$ along essential disks, a process which produces 3-balls, handlebodies, and compression bodies with only one interior boundary component, this is impossible. This proves Claim 3.

Let $\tilde{T}$ be a component of $V_{1}^{*}-C$ (for $C$ as above). Then $\partial \tilde{T}$ is a torus, so, by the same reasoning as above, and Claims 1,2 and $3, \tilde{T}$ must be a solid torus. It follows that $\partial \tilde{T}$ is comprised of narrow annuli and, by Claims 1, 2, and 3, pairs of adjacent wide annuli.

Let $\tilde{A}$ be a subannulus of $A$ connecting $\partial \tilde{T}$ and $\partial C$. We may assume, by rechoosing $\tilde{T}$ to be outermost if necessary, that $\tilde{A} \subset V_{2}$. It follows from the preceeding paragraph that $\tilde{T}$ has a meridian disk $D$ consisting of boundary reducing disks in the subtori cut off by narrow annuli and of disks (rectangles) defining a parallelism between the pairs of wide annuli. Now $\#|\partial D \cap \tilde{A}|=1$. Hence the requirements of Definition 14 are met. So the splitting surface of the Heegaard splitting $\left(\tilde{V}_{1}, \tilde{V}_{2}\right)$ obtained by replacing the appropriate arc in the tunnel system under consideration into its dual intersects $A$ in two fewer essential curves. It thus corresponds to a tunnel system which violates the minimality assumptions.

Theorem 12. Let $K_{1}$ and $K_{2}$ be small knots. Suppose $K_{1} \# K_{2}$ satisfies Assumption 1 and the tunnel system $\mathcal{T}$ for $K_{1} \# K_{2}$ satisfies Assumption 2. Then $t\left(K_{1}\right)+t\left(K_{2}\right)-1 \leq t\left(K_{1} \# K_{2}\right)$.

Proof. Let $\left(V_{1}, V_{2}\right)$ be the Heegaard splitting corresponding to the tunnel system for $K_{1} \# K_{2}$. Isotope the splitting surface $F$ of $\left(V_{1}, V_{2}\right)$ so that it intersects the decomposing annulus $A$ for $K_{1} \# K_{2}$ only in essential circles and so that $|F \cap A|$ is minimal.

Case 1: $|F \cap A|=2$.

Cut $C\left(K_{1} \# K_{2}\right)$ along $A$ and set $V_{1}^{i}=\left(V_{1} \cap C\left(K_{i}\right)\right) \cup \operatorname{collar}\left(A \cap V_{2}\right)$, and let $V_{2}^{i}$ be the closure of the complement of $V_{1}^{i}$ in $C\left(K_{i}\right)$. 


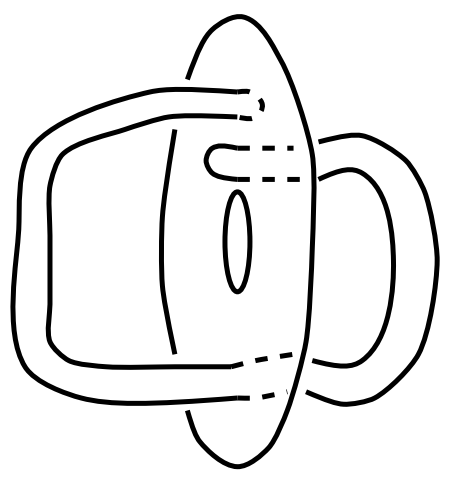

A component of $\mathrm{V}_{1}^{*}$ intersecting $\mathrm{A}$

Figure 4 .

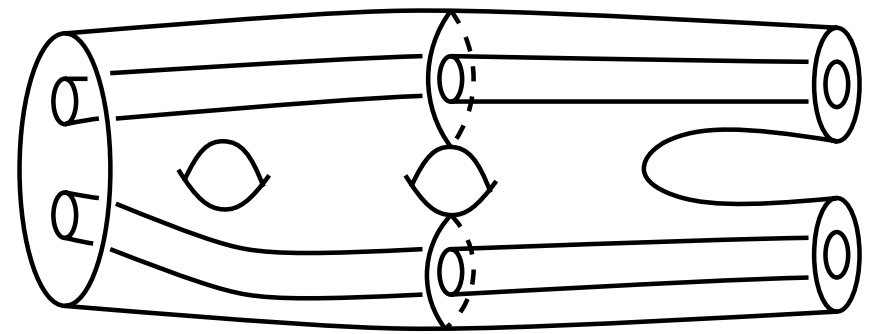

$\mathrm{V}_{1}$ before cutting along A

Figure 5a.

Claim: $\left(V_{1}^{i}, V_{2}^{i}\right)$ is a Heegaard splitting for $C\left(K_{i}\right)$. (See fig. 5a and fig. 5b)

Let $\mathcal{D}_{1}$ be a defining collection of disks for $V_{1}$. After isotopy, we may assume that $\mathcal{D}_{1} \cap A=\emptyset$. Then $\mathcal{D}_{1}^{i}=\mathcal{D}_{1} \cap M^{i}$ cuts $V_{1}^{i}$ into (closed surface) $\times I$. (Here $\mathcal{D}$ cuts $V_{1}$ into $\partial_{-} V_{1}$; then cutting $\partial_{-} V_{1}$ along $A^{\prime} \sqcup A^{\prime \prime}$ and adding the collar creates (closed surface) $\times I$.) Hence $V_{1}^{i}$ is a compression body.

$V_{2}^{i}$ is a compression body by Lemma 2 .

Let $g^{i}$ be the genus of $\left(V_{1}^{i}, V_{2}^{i}\right)$ and $g$ the genus of $\left(V_{1}, V_{2}\right)$. Then, by an Euler characteristic argument, $g^{1}+g^{2}=g+1$. Here the spine of $V_{1}^{i}$ defines a tunnel system corresponding to the Heegaard splittings $\left(V_{1}^{i}, V_{2}^{i}\right)$, hence $t\left(K_{1}\right)+t\left(K_{2}\right) \leq$ $t\left(K_{1} \# K_{2}\right)$.

Case 2: $|F \cap A|=4$. 

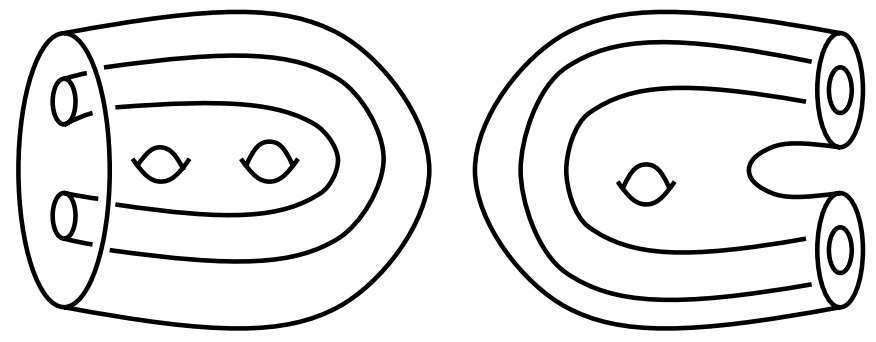

$\mathrm{V}_{1}^{1}$ and $\mathrm{V}_{1}^{2}$

Figure 5b.

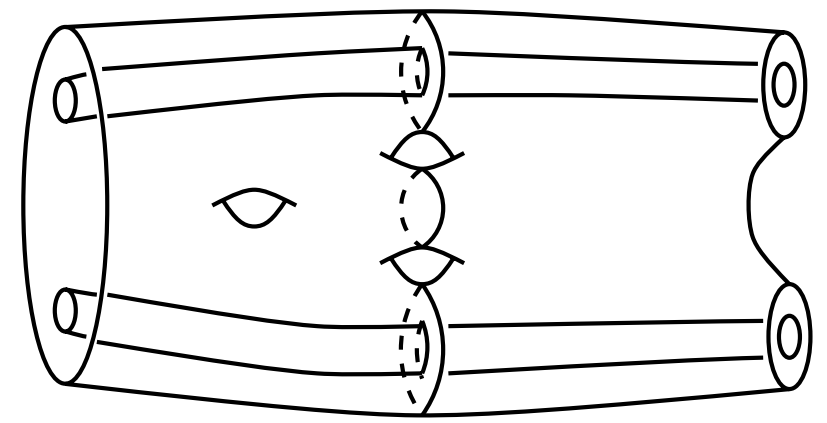

$\mathrm{V}_{1}$ before cutting along $\mathrm{A}$

Figure 6a.

As in the proof of Lemma 11, one of the components of $V_{1} \cap A$ is boundary reducible in $V_{1}$. We may assume, by choosing an outermost one, that the boundary reducing disk is disjoint from $A$. Then after performing the boundary compression along this disk, $V_{1} \cap A$ consists of two annuli and one disk, and $V_{2} \cap A$ is a pair of pants.

As before, cut $C\left(K_{1} \# K_{2}\right)$ along $A$ and set $V_{1}^{i}=\left(V_{1} \cap C\left(K_{i}\right) \cup\left(\operatorname{collar}\left(V_{2}\right.\right.\right.$ $\cap A)$ ) and $V_{2}^{i}$ equal to the closure of the complement of $V_{1}^{i}$ in $C\left(K_{i}\right)$. (See fig. 6a and fig. 6b.) Then by an argument similar to the one above (add the disk $D$ to $\mathcal{D}_{1}^{i}$ as necessary), $\left(V_{1}^{i}, V_{2}^{i}\right)$ is a Heegaard splitting of $M^{i}$. By the same reasoning as above, we find that $t\left(K_{1}\right)+t\left(K_{2}\right)-1 \leq t\left(K_{1} \# K_{2}\right)$.

By Lemma 11, this completes the proof.

Corollary 13. Let $K_{1}$ and $K_{2}$ be small knots, then $t\left(K_{1}\right)+t\left(K_{2}\right)-1 \leq t\left(K_{1}\right.$ $\left.\# K_{2}\right)$. 


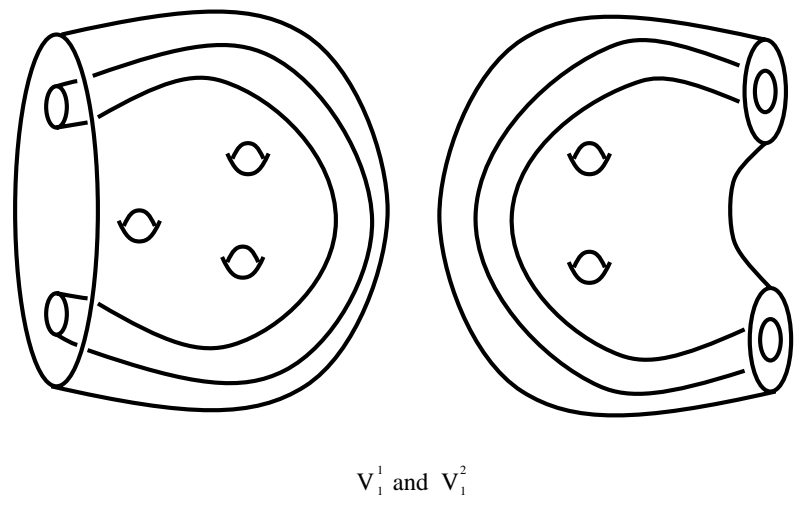

Figure 6b.

Proof. This follows from Theorem 9 and Theorem 12.

\section{Simultaneous decomposing annuli}

The arguments in the two preceeding sections can be tailored to show that for small knots $K_{1}, \ldots, K_{n}, t\left(K_{1} \# \ldots \# K_{n}\right) \geq n$. In the following we will assume that the decomposing annuli for $C\left(K_{1} \# \ldots \# K_{n}\right)$ have been chosen so that each $C\left(K_{i}\right)$ meets at most two decomposing annuli (i.e., we assume that the decomposing spheres are nested in "Matryoshka" fashion).

Lemma 14. Let $S$ be a separating essential surface in $C\left(K_{1} \# \ldots \# K_{n}\right)$, where each $K_{i}$ is a small knot. Then, after isotopy, for any $i, S \cap C\left(K_{i}\right)$ is either empty, or boundary parallel; i.e., $S$ is a swallow follow torus.

Proof. Isotope $S$ so that $\#|S \cap \mathcal{A}|$ is minimal. Let $A^{1}, \ldots, A^{n-1}$ be the decomposing annuli for $C\left(K_{1} \# \ldots \# K_{n}\right)$. Consider $C\left(K_{i}\right)$ and suppose it is cut off of $C\left(K_{1} \# \ldots \# K_{n}\right)$ by $A^{i}$, and perhaps $A^{i+1}$. If $S$ does not intersect $A^{i}$ or $A^{i+1}$, for any $l$, then $S$ must be disjoint from $C\left(K_{i}\right)$, since $K_{i}$ is small. If $S$ intersects $A^{i}$ or $A^{i+1}$, then $S$ must be boundary parallel in $C\left(K_{i}\right)$ to avoid the contradiction in the proof of Lemma 8.

Theorem 15. Let $K_{1}, \ldots, K_{n}$ be small knots, then $t\left(K_{1} \# \ldots \# K_{n}\right) \geq n$.

Proof. Suppose the inequality does not hold, and let $K_{1}, \ldots, K_{n}$ be small knots chosen so that $n$ is minimal among such collections of small knots. Let $\left(V_{1}, V_{2}\right)$ be the Heegaard splitting corresponding to a tunnel system $\mathcal{T}$ which realizes the $t\left(K_{1} \# \ldots \# K_{n}\right)$. 


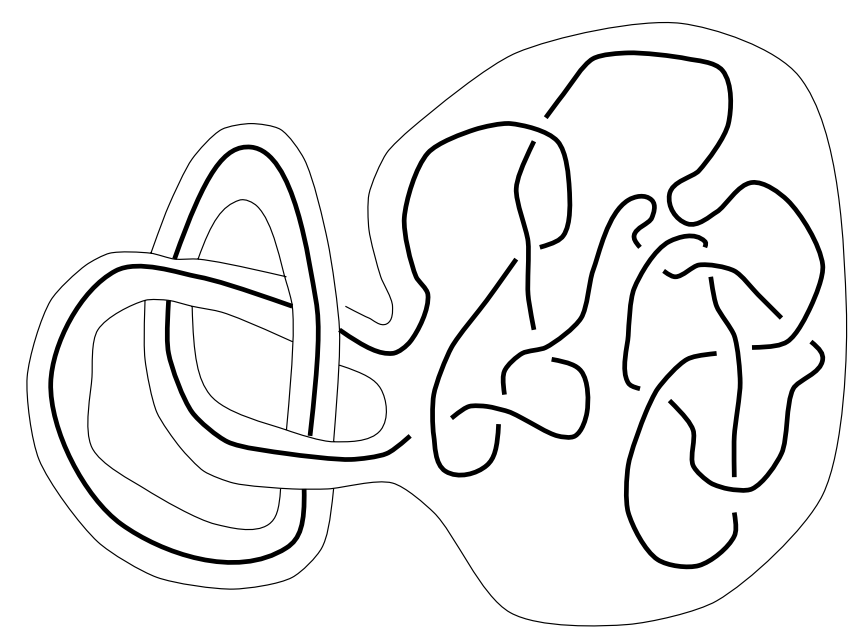

A generalized swallow-follow torus

Figure 7.

Claim 1: $\left(V_{1}, V_{2}\right)$ is strongly irreducible.

Suppose $\left(V_{1}, V_{2}\right)$ is weakly reducible. Since $\mathcal{T}$ realizes $t\left(K_{1} \# \ldots \# K_{n}\right)$, $\left(V_{1}, V_{2}\right)$ is irreducible. Consider an untelescoping of $\left(V_{1}, V_{2}\right)$. By Remark $7, k$ $\geq 1$. Again, if an $S^{i}$ were boundary parallel in $C\left(K_{1} \# \ldots \# K_{n}\right)$, then the untelescoping of $\left(V_{1}, V_{2}\right)$ would define a Heegaard splitting of $C\left(K_{1} \# \ldots \# K_{n}\right)$ of lower genus, contradicting the choice of $\mathcal{T}$. Hence each $S^{i}$ must be a generalized swallow follow torus. A generalized swallow follow torus separates $C\left(K_{1} \# \ldots\right.$ \# $\left.K_{n}\right)$ into $C\left(K_{i_{1}} \# \ldots \# K_{i_{j}}\right)$ minus a 2 -handle and $C\left(K_{i_{j+1}} \# \ldots \# K_{i_{n}}\right)$ for some partition $\left\{i_{1}, \ldots, i_{j}\right\} \sqcup\left\{i_{j+1}, \ldots, i_{n}\right\}$ of $\{1, \ldots, n\}$. Thus the untelescoping provides at least one Heegaard splitting corresponding to tunnel systems violating the choice of $K_{1}, \ldots K_{n}$.

In the following, we assume that the splitting surface of $\left(V_{1}, V_{2}\right)$ has been isotoped to intersect the decomposing annuli only in essential curves, and in the least number of such curves.

Claim 2: $\mathcal{T}$ can be chosen so that the splitting surface $F$ of $\left(V_{1}, V_{2}\right)$ intersects at most one of the decomposing annuli in more than 2 curves and it intersects this decomposing annulus in exactly 4 curves.

The proof of this Claim mimics and extends the proof of Lemma 11. Suppose the Claim is not true. Proceed as in the proof of Lemma 11, performing one boundary compression to produce a disk in the intersection of $V_{1}$ with one of the decomposing annuli, then performing an ambient 2-surgery on $F$ along this disk, and then performing ambient 2 -surgeries on all compressing disks for the resulting 
surface which are disjoint from all the decomposing annuli, to obtain a surface $F^{*}$, each component of which is separating. Again, denote the sides of $F^{*}$ by $V_{1}^{*}$ and $V_{2}^{*}$. Again, $V_{1}^{*} \subset V_{1}$ and $V_{2}^{*}$ is connected. Again, a component of $F^{*} \cap$ $C\left(K_{i}\right)$ is boundary parallel. Such a component could either be a narrow annulus as in the proof of Lemma 11, or a wide annulus, but here a wide annulus may either have boundary components on the same decomposing annulus, or on distinct decomposing annuli. Other possibilities would make the meridian a boundary slope and produce the same contradiction as before. Claims 1, 2, and 3 in the proof of Lemma 11 still hold.

Denote the component of $V_{1}^{*}$ containing $\partial C\left(K_{1} \# \ldots \# K_{n}\right)$ by $C$, and consider a component $T$ of $V_{1}^{*}-C$. As before, $T$ must be a solid torus. A meridian of $T$ can be constructed as before. Again we obtain a new tunnel system $\mathcal{T}^{\prime}$ for $K_{1} \# \ldots \# K_{n}$, by changing an arc of $\mathcal{T}$ into its dual, which contradicts the minimality assumptions on $\mathcal{T}$. This proves the Claim.

The arguments in Theorem 12 now only give the weaker result: $t\left(K_{1} \# \ldots\right.$ \# $\left.K_{n}\right) \geq n-1$. However, applying the argument in Case 1 of Theorem 12 along all decomposing annuli which intersect $F$ exactly twice, and noting that the remaining composite summand has tunnel number at least 2, since tunnel number 1 knots are prime (see for instance [11]), proves the Theorem.

\section{References}

[1] F. Bonahon, J. P. Otal, Scindements de Heegaard des Espaces Lenticulaires, Ann. scient. Ec. Norm. Sup. $4^{e}$ série, t. 16, 451-466.

[2] A. J. Casson, C. McA. Gordon, Reducing Heegaard splittings, Topolgy Appl. 27 (1987), $275-283$.

[3] M. Culler, C. Gordon, J. Luecke, P. Shalen, Dehn surgery on knots, Annals of Mathematics 125 (1987), 237-300.

[4] W. Jaco, Lectures on Three manifold Topology, CBMS Regional Conference Series in Mathematics 43 (Conference Board Mathematical Science, Washington, DC, 1980).

[5] R. Kirby, Problem's in Low-Dimensional Topology, preprint.

[6] T. Kobayashi, A construction of arbitrary high degeneration of tunnel number of knots under connected sum, J. Knot Theory Ramifications 3(2) (1994), 179-186.

[7] T. Kobayashi, Non-separating incompressible tori in 3-manifolds, J. Math. Soc. Japan 36 (1984), 11-22.

[8] Y. Moriah, H. Rubinstein, Heegaard Structures of Negatively Curved 3-Manifolds, preprint.

[9] K. Morimoto, On the additivity of tunnel number of knots, Topology Appl. 5 (1993), 37-66.

[10] K. Morimoto, M. Sakuma, Y. Yokota, Examples of tunnel number one knots which have the property " $1+1=3$ " Math. Proc. Cambridge Philos. Soc. 119(1) (1996), 113-118.

[11] M. Scharlemann, Tunnel number one knots satisfy the Poenaru conjecture, Topology Appl. 18 (1984), 235-258.

[12] M. Scharlemann, Heegaard splittings of compact 3-manifolds, preprint.

[13] M. Scharlemann, A. Thompson, Thin position for 3-manifolds, AMS Contemporary Math. 164 (1994), 231-238.

[14] M. Scharlemann, A. Thompson, Heegaard splittings of (surface) $\times$ I are standard, Math. Ann. 295 (1993), 549-564.

[15] J. Schultens, The classification of Heegaard splittings for (compact orientable surface) $\times$ 
$\mathrm{S}^{1}$, Proc. London Math. Soc. 67(3) (1993), 425-448.

[16] J. Schultens, Heegaard splittings of Seifert fibered spaces with boundary, Trans. AMS 347(7) (1995), 2533-2552.

Jennifer Schultens

Department of Mathematics

University of California

Berkeley, CA 94720

USA

e-mail: jcs@math.berkeley.edu

(Received: March 10, 1997) 